\title{
A Case of Cefoperazone/Sulbactam Induced Toxic Epidermal Necrolysis in a Middle Aged Egyptian Female.
}

\author{
Ahmed Elmazaly ${ }^{1}$ and Manar Mohamed ${ }^{1}$ \\ ${ }^{1}$ Ain Shams University Faculty of Medicine
}

September 16, 2020

\begin{abstract}
Background: Toxic Epidermal Necrolysis (TEN) is a rare, drug induced life-threatening mucocutaneous condition caused by immune system activation. TEN is associated with widespread keratinocyte death causing full-thickness denudation of the skin and mucosa, resulting in sepsis and a mortality rate of 30\%. Case Description: A 32-year-old female patient was precribed cefoperazone/sulbactam for sore throat. One day later she experienced an itchy rash on her hands. She was given IM dexamethazone for 4 days with no improvement. She presented to the emergency department 6 days later with severe itchy rash. On examination, there was a generalized symmetrical, desquamating erythematous rash distributed on the face, chest and back, with buccal ulceration and violaceous discoloration of the hands and feet. Vital signs were normal. Routine laboratory investigations were significant for high C-reactive protein and low serum albumin. Serology for HIV was negative. A diagnosis of cefoperazone/sulbactam-induced-TEN was made and the drug was discontinued. Evaluation of the SCORE of TEN (SCORTEN) on day one was 1 . The patient was admitted to the intensive care unit where she received supportive treatment and intravenous immunoglobulin (IVIG) at a dose of $2 \mathrm{gm} / \mathrm{kg}$ over 5 days with gradual improvement. Discussion: Cefoperazone/Sulbactaminduced-TEN is an idiosyncratic, dose independent adverse event. IvIG was used 7 days after appearance of the desquamation with considerable recovery. Keywords: Cefoperazone/Sulbactam Stevens - Johnson syndrome, Toxic Epidermal Necrolysis.
\end{abstract}

\section{Introduction}

Toxic epidermal necrolysis (TEN) and Steven-Johnson syndrome (SJS) are rare but life-threatening mucocutaneous conditions induced by immune system activation mostly caused by certain drugs and their metabolites, Associated with widespread keratinocyte death causing full-thickness denudation of the skin and mucosa, which results in a high susceptibly to sepsis with a mortality rate of $30 \%(\mathbf{1})$. The cutaneous and mucosal manifestations are commonly preceded by many non-specific symptoms including fever, rhinitis, headache, conjunctivitis and sore throat lasting approximately one week . Complications include infections, eye involvement (potentially leading to blindness), scars involving the mouth, pharynx, oesophagus, rectus, middle airways and genitourinary tract (phimosis, vaginal stenosis, dysuria) . Other deadly complications include renal and/or hepatic failure, dehydration, and sepsis (2) . There are two entities of the same clinical condition differing only in their percentage involved in their body surface. The percentage of the body surface involved is the only difference between SJS and TEN. Where, body surface area $(<10 \%)$ involvement is reported in SJS, 10-30\% BSA in SJS-TEN overlap and $>30 \%$ BSA detachment is reported in TEN(3) . The most common trigger for TEN/SJS are medications and usually triggers the disease within 8 weeks in both adults and children; however, the typical exposure period ranges from 4 days to 4 weeks. Commonly accused medications include non-steroidal anti-inflammatories, allopurinol, anticonvulsants such as lamotrigine, phenytoin and carbamazepine, antibacterial sulphonamides and the antiretroviral nevirapine (3) .

\section{Case Presentation}


A 32-year-old female patient complained of sore throat and was prescribed IM cefoperazone/sulbactam 1500 mg. One day post-initiation, the patient started to complain of itchy rashes over the hands, she sought medical advice, Cefoperazone/sulbactam was immediately discontinued and was prescribed conservative treatment in the form of IM dexamethasone every 12 hours. 6 days post-initiation, the patient presented to ER with sever itchy rash. On examination she had a generalized symmetrical, erythematous rash more marked on the face, upper limbs, chest and back with violaceous colour of both hands and feet. In addition, she had an associated buccal ulceration and conjunctival injection. Her temperature was $37^{\circ} \mathrm{C}$, with $\mathrm{SpO} 2$ of $98 \%$, a blood pressure recording of $100 / 60 \mathrm{mmHg}$ and a regular pulse of 80 beats/min. Vesiculobullous lesions were observed over the course of the admission, with sloughing of skin, especially on the chest and face around the lips. Routine laboratory assessments showed normal blood counts (7500 cells/uL) without hyper eosinophilia (0.02\%). Hepatic enzyme levels, renal function, and serum electrolyte levels were all within normal limits and a serological test was negative for HIV. CRP was high $(55 \mathrm{mg} / \mathrm{l})$ with low serum Albumin $(3.1 \mathrm{~g} / \mathrm{dl})$. A diagnosis of TEN secondary to cefoperazone/sulbactam exposure was made. Evaluation of the SCORE of Toxic Epidermal Necrosis (SCORTEN) score on day 1 indicated a score of 1 . The patient was admitted to intensive care unit and received intravenous fluid resuscitation and urinary catheter was inserted. Blood and urine cultures were conducted. Dermatological, ophthalmological and burn unit consultation were done. Skin punch biopsy was done which confirmed the diagnosis of TEN. Supportive treatment was formed; the patient was kept warm, had careful protection of the eroded areas, tetracycline eye ointment, antiseptic mouth wash and both Intravenous and non-intravenous hydration. Immunosuppressive treatment was initiated on the second day of admission; IvIG $2 \mathrm{gm} / \mathrm{kg}$ divided by 5 days by infusion over 6 hours. The patient recovered considerably after 5 days of immunosuppressive, symptomatic and supportive management and was transferred to the ward for further follow up and discharged and was asked to follow up at OPC basis.

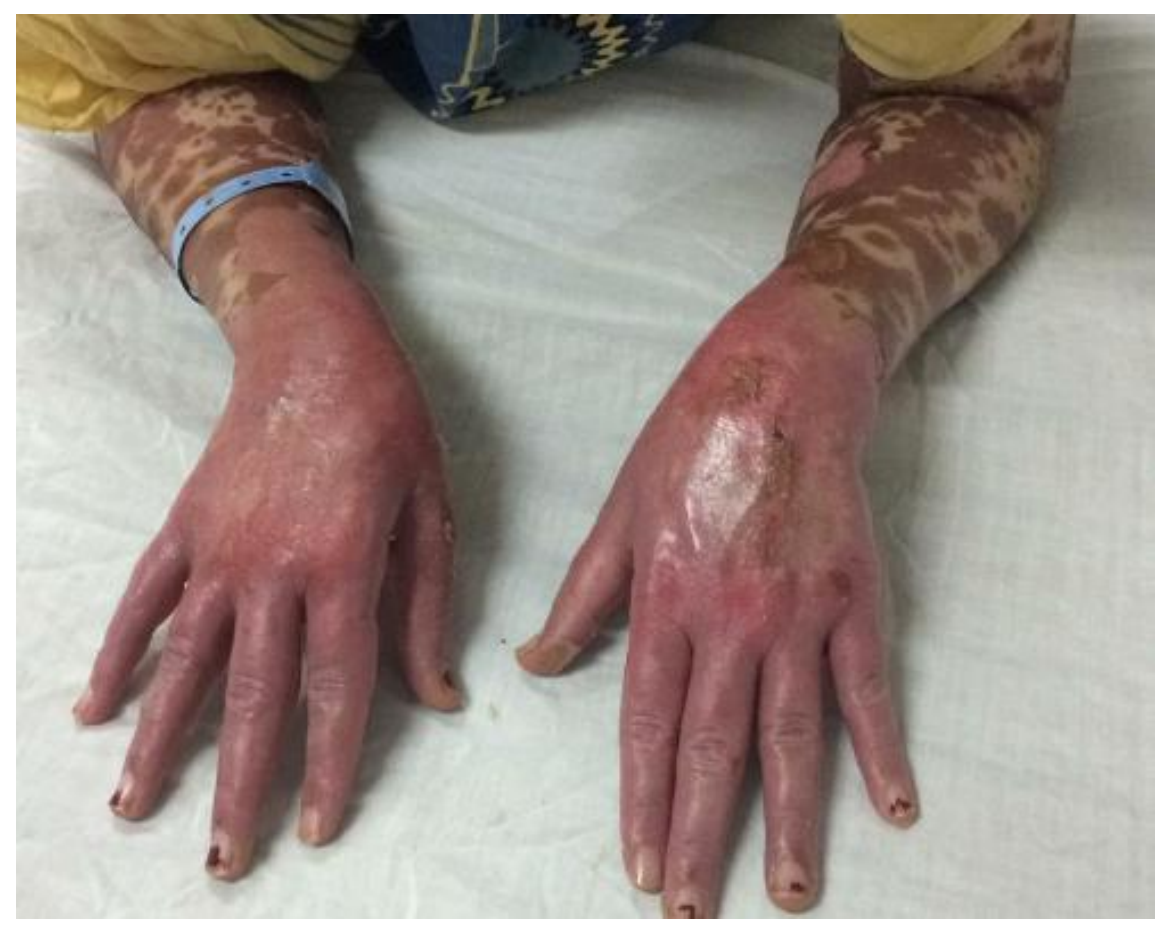




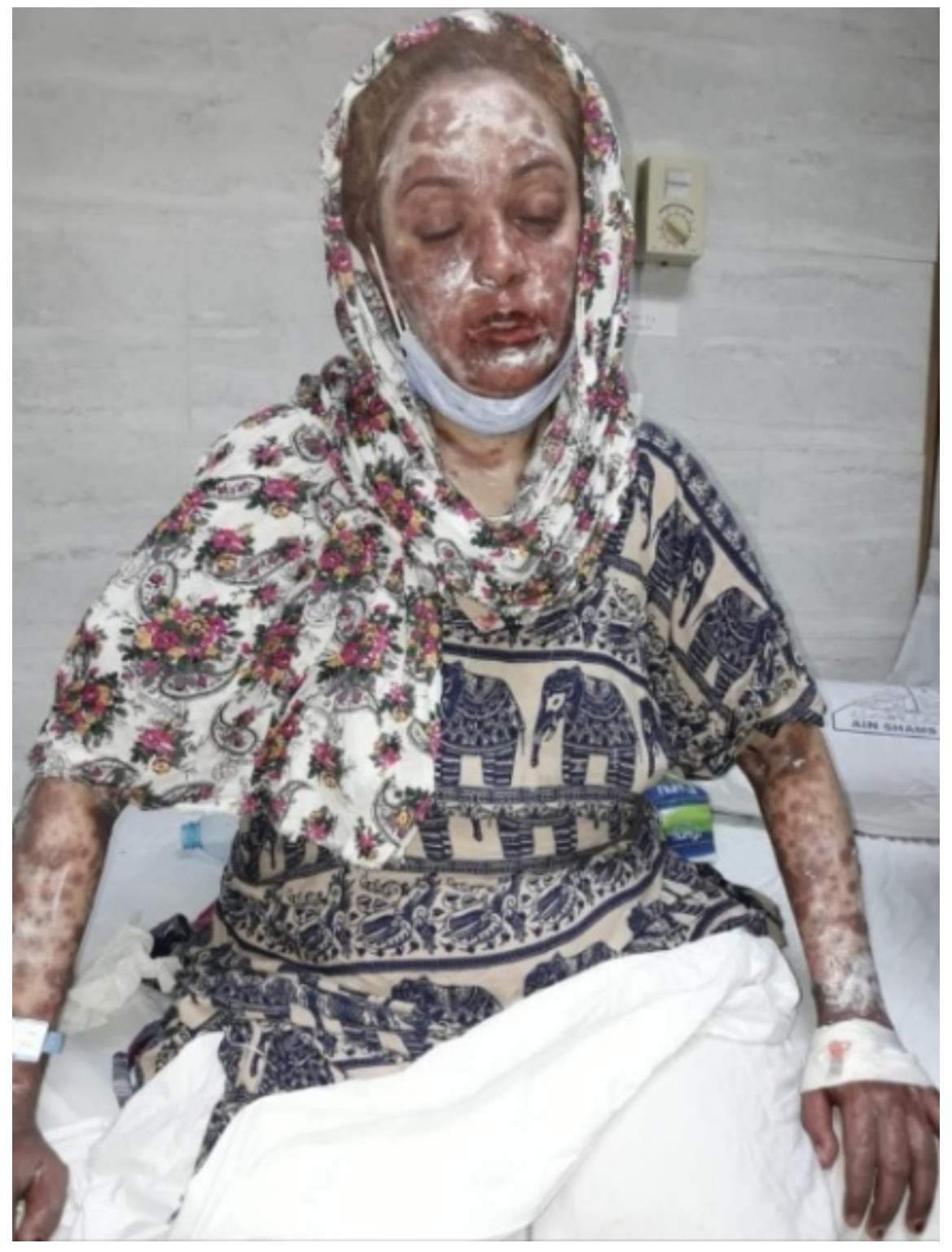




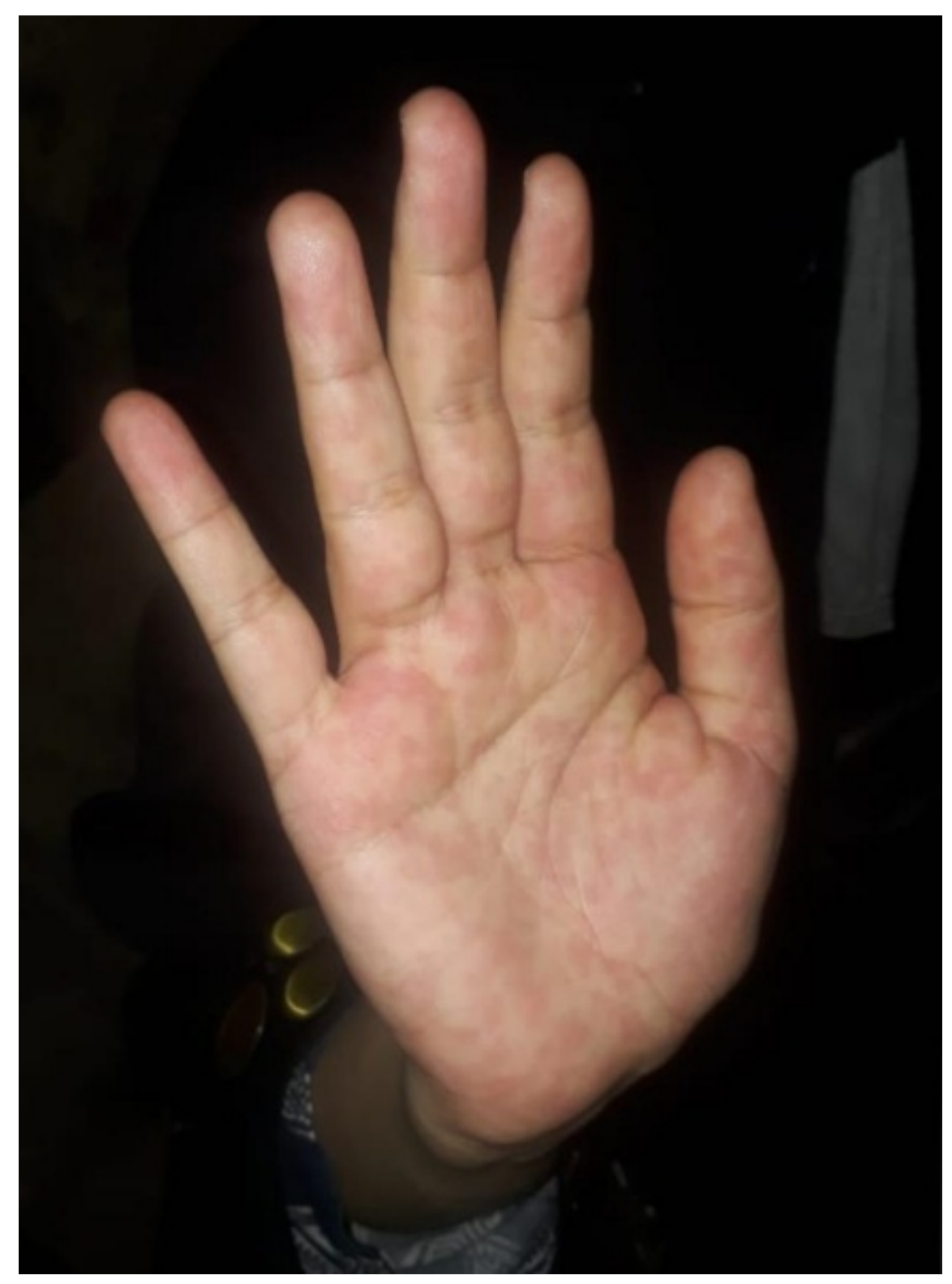

Day (1) post-initiation Day (7) post-initiation Day (12) post-initiation

\section{Discussion}

Cefoperazone/sulbactam-induced SJS/TEN is mostly an idiosyncratic, dose independent, unpredictable adverse event (4). But there are important factors such as the individual variation in drug metabolism or clearance, HIV-1 seropositivity, polypharmacy and competitive drug inhibition may also play role in cefoperazone/sulbactam induced SJS/TEN(5). The mortality risk of patients in TEN is around 40\%. High dose IVIG therapy given in early SJS and TEN has been beneficial to arrest disease progression and reduce mortality, although not f. Doses at [?]2 $\mathrm{g} / \mathrm{kg}$ were associated with less mortality. IVIG treatment should be initiated early after the diagnosis. The use of corticosteroids and immunosuppressive agents simultaneous to IVIG therapy is controversial. The dose of IVIG in TEN is advised to be a total of [?] $3 \mathrm{~g} / \mathrm{kg}$ divided into 3-5 days at a time. Long term treatment is not recommended(6) . 


\section{Conclusion}

Cefoperazone/sulbactam-induced-TEN is an idiosyncratic, dose independent adverse event. IvIG was used 7 days after appearance of the desquamation with considerable recovery.

\section{Acknowledgments}

Allergy \& Clinical Immunology Division, Ain Shams University

\section{Ethical Consideration}

Informed oral \& written consent was provided.

\section{References}

1- Charlton OA, Harris V, Phan K, Mewton E, Jackson C and Cooper A. Toxic Epidermal Necrolysis and Steven-Johnson Syndrome: A Comprehensive Review Advances in Wound Care 2020 9:7, 426-439.

2- De Guido C, Calderaro A, Ruozi MB., et al. An unusual cause of Steven-Johnson Syndrome. Acta Bio-medica : Atenei Parmensis. 2020 Mar;91(1):128-131.

3- Naga Subrahmanyam S, Nagaraju G V and Tagoore Vijaya Lakshmi D. Phenytoin induced Steven Johnson syndrome. International Journal of Research in Pharmaceutical Sciences. 2020;11(1), 173175 .

4- Johnston A and Uetrecht J. Current understanding of the mechanism of idiosyncratic drug-induced agranulocytosis. Expert Opin Drug Metab Toxicol. 2015;11(2):243-57.

5- Dodiuk-Gad RP, Laws PM and Shear NH. Epidemiology of severe drug hypersensitivity. Semin Cutan Med Surg. 2014;33(1):2-9.

6- Emre S. Intravenous immunoglobulin treatment: Where do dermatologists stand? Dermatologic Therapy. 2019; 32:e12854. 